\title{
SOME THEOREMS ON A GENERALIZED LAPLACE TRANSFORM OF GENERALIZED FUNCTIONS
}

\author{
AWADHESH CHANDRA GUPTA AND ANIL KUMAR MAHATO
}

\begin{abstract}
In this paper we extend the generalized Laplace transform $F(s)=$ $\frac{\Gamma(\beta+\eta+1)}{\Gamma(\alpha+\beta+\eta+1)} \int_{0}^{\infty}(s t)^{\beta}{ }_{1} F_{1}(\beta+\eta+1, \alpha+\beta+\eta+1 ;-s t) f(t) d t$ where $f(t) \in L(0, \infty)$, $\beta \geq 0, \eta>0$; to a class of generalized functions. We will extend the above transform to a class of generalized functions as a special case of the convolution transform and prove an inversion formula for it.
\end{abstract}

\section{Introduction}

A generalization of the Laplace transform

$$
F(s)=\int_{0}^{\infty} e^{-s t} f(t) d t
$$

is given by Joshi [5]:

$$
F(s)=\frac{\Gamma(\beta+\eta+1)}{\Gamma(\alpha+\beta+1)} \int_{0}^{\infty}(s t)_{1}^{\beta} F_{1}(\beta+\eta+1, \alpha+\beta+\eta+1 ;-s t) f(t) d t .
$$

Recently Gupta and Mahato [2] extended the transform (1.2) to a class of generalized functions and an analyticity theorem is proved for it. In Gupta and Mahato [3], a complex inversion formula for (1.2) has been extended to a class of generalized functions.

In this paper we discuss the generalized Laplace transform (1.2) as a special case of the convolution transform

$$
F(x)=\langle f(t), G(x-t)\rangle .
$$

We also prove an inversion formula for (1.2) in the distributional sense.

\section{The testing function spaces $D, D(I), L_{c, d}, J_{c, d}$ and their duals}

Received May 20, 1993; revised October 15, 1993

1991 Mathematics Subject Classification. 44; 46F12.

Key words and phrases. Generalized Laplace transform, testing function space, distribution. 
A function is said to be smooth if its derivatives of all orders are continuous at all points of its domain. The space $D$ consists of all complex-valued functions $\phi$ that are smooth zero outside some finite interval. Let $I$ be the interval $0<t<\infty$. $D(I)$ is the space of infinitely differentiable functions having compact supports defined over the interval $I$.

Let $\lambda_{c, d}(t)$ be the function:

$$
\lambda_{c, d}(t)= \begin{cases}e^{c t}, & 0 \leq t<\infty \\ e^{d t}, & -\infty<t<0 .\end{cases}
$$

$L_{c, d}$ denotes the space of all complex-valued smooth functions $\phi(t)$ on $-\infty<t<\infty$ on which the functionals defined by

$$
\gamma_{k}(\phi) \triangleq \gamma_{c, d, k}(\phi)=\sup _{-\infty<t<\infty}\left|\lambda_{c, d}(t) D^{k} \phi(t)\right|
$$
assume finite values. We assign to $L_{c, d}$ the topology generated by $\left\{\gamma_{k}\right\}_{k=0}^{\infty}$ thereby
making it a countably multinormed space.

Applying the change of variable

$$
T=e^{-t}, t=(-\log T), D_{t}=\left(-T D_{T}\right)
$$

to the definition of $L_{c, d}$ and setting $T \psi(T)=\phi(-\log T)$ in (2.2), we have the following definition:

Given any two real numbers $c$ and $d, J_{c, d}$ is the space of all smooth functions $\psi(T)$ on $0<T<\infty$ such that

$$
\begin{aligned}
i_{k}\{\psi(T)\} & \triangleq i_{c, d, k}\{\psi(T)\} \\
& =\sup _{0<T<\infty}\left|\lambda_{c, d}(-\log T)\left(-T D_{T}\right)^{k}\{T \psi(T)\}\right| \\
& <\infty, k=0,1,2, \ldots
\end{aligned}
$$

where

$$
\lambda_{c, d}(-\log T)=\left\{\begin{array}{l}
T^{-c}, 0<T \leq 1 \\
T^{-d}, 1<T<\infty
\end{array}\right.
$$

The topology of $J_{c, d}$ is that generated by the multinorm $\left\{i_{c, d, k}\right\}_{k=0}^{\infty}$. As a consequence $J_{c, d}$ is a complete countably multinormed space.

$D^{\prime}, D^{\prime}(I), L_{c, d}^{\prime}$ and $J_{c, d}^{\prime}$ are the dual spaces corresponding to the spaces $D, D(I), L_{c, d}$ and $J_{c, d}$ respectively.

Theorem 2.1. The mapping

$$
\psi(T) \mapsto e^{-t} \psi\left(e^{-t}\right)=\phi(t)
$$


is an isomorphism from $J_{c, d}$ onto $L_{c, d}$.

The inverse mapping is given by

$$
\phi(t) \mapsto\left(T^{-1}\right) \phi(-\log T)=\psi(T)
$$

Proof. It is obvious that the mapping (2.5) and (2.6) are linear and inverses of one another.

Let $\psi(T) \in J_{c, d}$. Some computations show that $D_{t}^{k}\left\{e^{-t} \psi\left(e^{-t}\right)\right\}$ is equal to a finite sum of terms, a typical term being $a_{p} T^{p+1} D_{T}^{p} \psi(T)$, where $0 \leq p \leq k$ and $a_{p}$ is a constant. Thus,

$$
\begin{aligned}
\lambda_{c, d}(t) & D_{t}^{k}\left[e^{-t} \psi\left(e^{-t}\right)\right] \\
= & \sum_{p} a_{p} \lambda_{c, d}(-\log T) T^{p+1} D_{T}^{p} \psi(T) \\
= & \sum_{p} b_{p} \lambda_{c, d}(-\log T)\left(-T D_{T}\right)^{p}\{T \psi(T)\}
\end{aligned}
$$

(where $b_{p}$ is another constant) so that

$$
\begin{aligned}
\gamma_{c, d, k}(\phi) & =\gamma_{c, d, k}\left[e^{-t} \psi\left(e^{-t}\right)\right] \\
& \leq \sum_{p}\left|b_{p}\right| i_{c, d, p}\{\psi(T)\} .
\end{aligned}
$$

Consequently, (2.5) is a continuous mapping of $J_{c, d}$ into $L_{c, d}$. Now, let $\phi(t) \in L_{c, d}$. Again a straightforward computation shows that

$$
\left(-T D_{T}\right)^{k}\left[T T^{-1} \phi(-\log T)\right]=\sum_{p} C_{p} D_{t}^{p} \phi(t)
$$

where $0 \leq p \leq k$ and the $C_{p}{ }^{\prime} s$ are constants.

Therefore,

$$
\begin{aligned}
i_{c, d, k}(\psi) & =i_{c, d, k}\left[T^{-1} \phi(-\log T)\right] \\
& \leq \sum_{p}\left|C_{p}\right| \gamma_{c, d, p}(\phi) .
\end{aligned}
$$

Thus (2.6) is a continuous linear mapping of $L_{c, d}$ into $J_{c, d}$. Since the mapping (2.5) and (2.6) are one to one we can now conclude that they are also onto $L_{c, d}$ and $J_{c, d}$ respectively. Our proof is complete.

The dual space $L_{c, d}^{\prime}$ denotes the space of continuous linear functions on $L_{c, d}$. If $f(f) \in L_{c, d}^{\prime}$ we define $f(-\log T)$ as a functional on $J_{c, d}$ by

$$
\left\langle f(-\log T), T^{-1} \phi(-\log T)\right\rangle \triangleq\langle f(t), \phi(t)\rangle, \quad \phi(t) \in L_{c, d} .
$$


It can be easily proved that the mapping $f(t) \mapsto f(-\log T)$ defined by (2.9) is an isomorphism on $L_{c, d}^{\prime}$ onto $J_{c, d}^{\prime}$. The inverse mapping $f(T) \mapsto f\left(e^{-t}\right)$ is defined by

$$
\left\langle f\left(e^{-t}\right), \phi(t)\right\rangle \triangleq\langle f(T), \psi(T)\rangle
$$

\section{The generalized one-sided Laplace transformation}

Let $G(t)=\frac{\Gamma(A)}{\Gamma(B)} e^{\beta t}{ }_{1} F_{1}\left(A, B, e^{-t}\right) e^{t}$ where $A=\beta+\eta+1 ; B=\alpha+\beta+\eta+1$.

Setting $y=e^{x}, T=e^{-t}$ and $\phi(t)=G(x-t)$, we obtain

$$
\begin{aligned}
T^{-1} \phi(-\log T) & =e^{t} G(x-t) \\
& =\frac{\Gamma(A)}{\Gamma(B)} y(y T)^{\beta}{ }_{1} F_{1}(A, B ;-y T) .
\end{aligned}
$$

If we choose $c<1$ and $d$ as any real number, we may replace $\phi(t)$ by $G(x-t)$ in (2.9) to obtain

$$
\begin{aligned}
& \left\langle f(-\log T), \frac{\Gamma(A)}{\Gamma(B)} y(y T)^{\beta}{ }_{1} F_{1}(A, B ;-y T)\right\rangle \\
= & \langle f(t), G(x-t)\rangle \\
= & F(\log y) .
\end{aligned}
$$

Setting $J(y)=y^{-1} G(\log y)$ and $j(T)=f(-\log T)$ we finally obtain the new definition of the generalized one-sided Laplace transform

$$
J(y) \triangleq\left\langle j(T), \frac{\Gamma(A)}{\Gamma(B)}(y T)_{2}^{\beta} F_{1}(A, B ;-y T)\right\rangle, 0<y<\infty .
$$

This has a meaning as the application of $j(T) \in J_{c, d}^{\prime}$ to $\frac{\Gamma(A)}{\Gamma(B)}(y T)^{\beta}{ }_{2} F_{1}(A, B ;-y T) \in$ $J_{c, d}$ where $c<1$ and $d$ is arbitrary and positive.

Theorem 3.1. For any fixed real $y>0$,

$\frac{\Gamma(A)}{\Gamma(B)}(y T)^{\beta}{ }_{1} F_{1}(A, B ;-y T)$ is a member of $J_{c, d}$.

Proof. For $\frac{\Gamma(A)}{\Gamma(B)}(y T)^{\beta}{ }_{1} F_{1}(A, B ;-y T)$ to be in $J_{c, d}$ we have to show that

$$
\sup _{0<T<\infty}\left|\lambda_{c, d}(-\log T)\left(-T D_{T}\right)^{k}\{T \psi(T)\}\right|
$$

is bounded, where $\psi(T)=\frac{\Gamma(A)}{\Gamma(B)}(y T)^{\beta}{ }_{1} F_{1}(A, B ;-y T)$.

Now

$$
\begin{aligned}
& \sup _{0<T<\infty}\left|\lambda_{c, d}(-\log T)\left(-T D_{T}\right)^{k}\{T \psi(T)\}\right| \\
= & \sup _{0<T<\infty}\left|\lambda_{c, d}(-\log T)(-1)^{k} \sum_{p} T^{p+\beta+1} D_{T}^{p}\left\{-\frac{\Gamma(A)}{\Gamma(B)}{ }_{1} F_{1}(A, B ;-y T)\right\}\right|, 0 \leq p \leq k .
\end{aligned}
$$


By using the result of Erdelyi [1] p.254

$$
\frac{d^{n}}{d x^{n}}\left[{ }_{1} F_{1}(a ; c ; x)\right]=\frac{(a)_{n}}{(c)_{n}}{ }_{1} F_{1}(a+n, c+n ; x)
$$

where $(a)_{n}=a(a+1) \ldots(a+n-1)=\frac{\Gamma(a+n)}{\Gamma(a)}$.

Thus each side of (2.12) is equal to

$$
\sup _{0<T<\infty}\left|\lambda_{c, d}(-\log T)(-1)^{k} \sum_{p}(-1)^{p} T^{p+\beta+1} y^{p} \frac{\Gamma(A+p)}{\Gamma(B+p)}{ }_{1} F_{1}(A+p, B+p ;-y T)\right| .
$$

For $0<T \leq 1$, the above expression is

$$
\sup _{0<T \leq 1}\left|\sum_{p} T^{-c+p+\beta+1}(-1)^{k+p} y^{p} \frac{\Gamma(A+p)}{\Gamma(B+p)}{ }_{1} F_{1}(A+p, B+p ;-y T)\right| .
$$

From Slater [6], p.59, we have

$$
{ }_{1} F_{1}(a, b ;-x)=\frac{\Gamma(b)}{\Gamma(b-a)} x^{-a}\left\{1+O|x|^{-1}\right\}, x \rightarrow \infty
$$

and

$$
{ }_{1} F_{1}(a, b ;-x)=O(1), x \rightarrow 0 .
$$

Thus for $0<T \leq 1(2.13)$ is

$$
\begin{aligned}
& =\sup _{0<T \leq 1}\left|\sum_{p} T^{-c+p+\beta+1}(-1)^{k+p} y^{p} \frac{\Gamma(A+p)}{\Gamma(B+p)}\right| \\
& =\text { a finite quantity as }|T| \rightarrow 0 \text { if } 1-c>0 \text {, i.e. } c<1 .
\end{aligned}
$$

Now for $T \rightarrow \infty,(2.13)$ is

$$
\begin{aligned}
& =\sup _{0<T<\infty}\left|\sum_{p} T^{-d+p+\beta+1}(-1)^{k+p} y^{p} \frac{\Gamma(A)}{\Gamma(B)} \frac{\Gamma(\beta)}{\Gamma(\alpha)}(y T)^{-\beta-x-1-p}\right| \\
& =\sup _{0<T<\infty}\left|\sum \frac{\Gamma(A)}{\Gamma(\alpha)}(-1)^{k+p} T^{-d-n} y^{-\beta-\eta-1}\right| \\
& =\text { a finite quantity for any real positive value of } d .
\end{aligned}
$$

Thus, $\frac{\Gamma(A)}{\Gamma(B)}(y T)^{\beta}{ }_{1} F_{1}(A, B ;-y T)$ is a member of $J_{c, d}$ for every $c<1$ and $d>0$.

It can be easily proved that $J(y)$ is a smooth function on $0<y<\infty$. Thus if

$$
J(y)=\left\langle j(T),-\frac{\Gamma(A)}{\Gamma(B)}(y T)^{\beta}{ }_{1} F_{1}(A, B ;-y T)\right\rangle
$$


then

$$
J^{(n)}(y)=\left\langle j(T), \frac{\partial^{h}}{\partial y^{h}}\left\{-\frac{\Gamma(A)}{\Gamma(B)}(y T)^{\beta}{ }_{1} F_{1}(A, B ;-y T)\right\}\right\rangle
$$

where $n$ is a non-negative integer.

Theorem 3.2. Let $j(T)$ be an arbitrary element of $J_{c, d}^{\prime}$ and

$$
J(y)=\left\langle j(T),-\frac{\Gamma(A)}{\Gamma(B)}(y T)_{2}^{\beta} F_{1}(A, B ;-y T)\right\rangle .
$$

Then

$$
j(y)=O\left[y^{-p-\eta-1}\right] \quad \text { as } \quad y \rightarrow \infty .
$$

Proof. Using the boundedness property of generalized functions we get

$$
J(y) \leq C \max _{0<M<V} \sup _{0<T<\infty}\left|\lambda_{c, d}(-\log T)\left(-T D_{T}\right)^{M}\{T \psi(T)\}\right|
$$

where $\psi(T)=\frac{\Gamma(A)}{\Gamma(B)}(y T)^{\beta}{ }_{1} F_{1}(A, B ;-y T)$.

For $1<T<\infty$,

$$
\begin{aligned}
& \lambda_{c, d}(-\log T)\left(-T D_{T}\right)^{M}\left\{T \frac{\Gamma(A)}{\Gamma(B)}(y T)^{\beta}{ }_{1} F_{1}(A, B ;-y T)\right\} \\
= & T^{-d}\left(-T D_{T}\right)^{M}\left\{T \frac{\Gamma(A)}{\Gamma(B)}(y T)^{\beta}{ }_{1} F_{1}(A, B ;-y T)\right\} \\
= & T^{-d}(-1)^{M} \sum_{0 \leq p<M} a_{p} T^{\beta+p+1} D_{T}^{p}\left\{\frac{\Gamma(A)}{\Gamma(B)}{ }_{1} F_{1}(A, B ;-y T)\right\}\left(a_{p} \text { being constant }\right) \\
= & (-1)^{M} T^{-d}\left[\sum_{p} a_{p} T^{p+\beta+1}(-1)^{p} y^{p} \frac{\Gamma(A+p)}{\Gamma(B+p)}{ }_{1} F_{1}(A+p, B+p ;-y T)\right] \\
= & \sum_{p}(-1)^{M+p} a_{p} T^{-d+p+\beta+1} y^{p} \frac{\Gamma(A+p)}{\Gamma(B+p)}{ }_{1} F_{1}(A+p, B+p ;-y T) \\
= & \sum_{p}(-1)^{M+p} a_{p} T^{-d+p+\beta+1} y^{p} \frac{\Gamma(A+p)}{\Gamma(B+p)} \frac{\Gamma(B+p)}{\Gamma(\alpha)}(y T)^{-\beta-\eta-1-p}
\end{aligned}
$$

(Slater [6], p.59)

$=\sum_{p}(-1)^{M+p} T^{-d-n} y^{-\beta-\eta-1} a_{p} \frac{\Gamma(A+p)}{\Gamma(\alpha)}$.

For large $T$ and $y>0$, we have $t T^{-d-\eta} y^{-\beta-\eta-1} \leq$ (const.) $y^{-\beta-\eta-1}$. This establishes that for large $T$,

$$
|J(y)| \leq \text { (const.) } y^{-\beta-\eta-1}
$$


So $J(y)=O\left[y^{-\beta-\eta-1}\right]$ as $y \rightarrow \infty$.

\section{Inversion formula}

The technique employed in finding the inversion formula is as given in Zemanian [7] p.229-226.

The conventional convolution transform is

$$
F(x)=\int_{-\infty}^{\infty} f(t) G(x-t) d t
$$

and the corresponding inversion function $E(x)$, which serves to invert the transform is defined by the equation

$$
[E(x)]^{-1}=\int_{-\infty}^{\infty} G(y) e^{-x y} d y
$$

This conventional convolution has been extended a certain class of generalized functions [Zemanian, 7, p.229-246] and their inversion formula has been proved to be still valid when the limiting operation in that formula is understood as weak convergence in the space $D^{\prime}$ of Schwartz distributions.

Setting $s=e^{x}$ and $t=e^{-t}$ in the conventional generalized Laplace transform

$$
F(s)=\frac{\Gamma(A)}{\Gamma(B)} \int_{0}^{\infty}(s t)^{\beta}{ }_{1} F_{1}(A, B ;-s t) f(t) d t .
$$

We obtain

$$
\begin{aligned}
F\left(e^{x}\right) & =\frac{\Gamma(A)}{\Gamma(B)} \int_{-\infty}^{\infty} e^{(x-t)^{\beta}}{ }_{1} F_{1}\left(A, B ;-e^{(x-t)}\right) f\left(e^{-t}\right) e^{-t} d t \\
\text { or } \quad e^{x} F\left(e^{x}\right) & =\frac{\Gamma(A)}{\Gamma(B)} \int_{-\infty}^{\infty} e^{(x-t)(\beta+1)}{ }_{1} F_{1}\left(A, B ;-e^{(x-t)}\right) f\left(e^{-t}\right) d t \\
\text { or } \quad J(x) & =\frac{\Gamma(A)}{\Gamma(B)} \int_{-\infty}^{\infty} e^{(x-t)(\beta+1)}{ }_{1} F_{1}\left(A, B ;-e^{(x-t)}\right) j(t) d t \\
\text { where } J(x) & =e^{x} F\left(e^{x}\right) \text { and } j(t)=f\left(e^{-t}\right) .
\end{aligned}
$$

As in Joshi [5] and using some results from Hirschman and Widder [4] (p.66), the inversion operator $E(D)$ is given by

$$
\begin{aligned}
& E(D)\left\{e^{x} F\left(e^{x}\right)\right\}=E(D)\{J(x)\} \\
= & \lim _{n \rightarrow \infty}(-1)^{n} n^{\alpha-\beta+x} e^{(n+\beta) x} D_{1}^{n} e^{-(\beta+\eta+1) x} D_{1}^{n} e^{-\alpha x} D_{1}^{-n} e^{(\alpha+\eta) x} F\left(e^{x}\right) \\
= & f\left(e^{-t}\right)
\end{aligned}
$$

where $D_{1}=\frac{d}{d e^{\pi x}}$. 
Returning to the original variable, we have

$$
\lim _{n \rightarrow \infty}(-1)^{n} \frac{\Gamma(n+\alpha) n^{\log x}}{\Gamma(n+\beta)(n+1)} s^{n+\beta} D^{n} S^{-(\beta+\eta+1)} D^{n} s^{-\alpha} D^{-n} s^{\alpha+\eta} F(s)=f(t) .
$$

This has a sense as a limit in $D^{\prime}(I)$. The change of variable we have used in (2.11) defines an isomorphism from $D^{\prime}$ onto $D^{\prime}(I)$.

In summary if $j \in J_{c, d}^{\prime}$ for some $c<1$ and $d>0$ and if $J(x)$ is defined by (2.11), (4.3) holds true in the sense of convergence in $D^{\prime}(I)$.

As a consequence of inversion formula, we have Theorem 4.1 (The Uniqueness Theorem).

Let $f \in L_{c, d}^{\prime}$ and $h \in L_{c, d}^{\prime}$. Also let $F(s)=\langle f(t), G(s-t)\rangle$ and $H(s)=\langle h(t), G(s-$ $t)\rangle$. If $F(x)=H(x)$ for all $x$, then $f=h$ in the sense of equality in $D^{\prime}$.

\section{Acknowledgements}

The authors are thankful to the referee for his valuable constructive suggestions.

\section{References}

[1] A. Erdelyi, Higher Transcendental Functions, Vol.I, McGraw Hill, 1953.

[2] A.C. Gupta, and A.K. Mahato, On a generalized Laplace Transform of Generalized Functions, communicated for publication in Mathematica Balkanica, Yugoslavia.

[3] A.C. Gupta, and A.K. Mahato, "Complex Inversion and Uniqueness Theorems for a Generalized Laplace Transform," Indag. Mathem., N.S., 2(3), 301-310, 1991.

[4] Hirschman and Widder, The Convolution Transform, Princeton University Press, 1955.

[5] J.M.C. Joshi, "On a Generalized Stieltjes Transform," Pacific Jour. Math., 14(1969), 969-976.

[6] L.J. Slater, Confluent Hypergeometric Functions, Cambridge University Press, 1960.

[7] A.H. Zemanian, Generalized Integral Transformation, Interscience Publ., New York, 1968.

Karam Chand Bhagat College, Bero, Ranchi-835202, India.

Marwari College, Ranchi-834001, India. 